\title{
Un nouveau support de communication : l'éco-oenotourisme, paysage, biodiversité, écoconception des caves
}

\author{
Joël Rochard ${ }^{\mathrm{a}}$ \\ IFV (Institut Français de la Vigne et du Vin), Pôle National Développement Durable, 17 rue Jean Chandon Moët, \\ 51200 Epernay, France
}

\begin{abstract}
Résumé. L'œnotourisme prend une part grandissante dans la valorisation des territoires viticoles. C'est également un facteur qui participe à l'image et à la valeur ajoutée des vins de ces régions. Parallèlement à l'attrait culturel pour la vigne, le vin et la dégustation, « l'Enotourisme » est souvent motivé par une approche écologique des caves et des terroirs. Ainsi émerge le concept 《d'Eco-Oenotourisme » qui associe notamment, en liaison avec le développement durable, les paysages, la biodiversité, ainsi que l'éco-conception des caves. Cette thématique est importante pour les viticulteurs qui sont sensibles au regard que la société porte sur leur métier, voire pour l'acceptation locale de leurs activités et des projets qu'ils souhaitent mettre en œuvre. Au-delà de l'approche purement descriptive, le paysage est l'expression esthétique de l'écosystème culturel que constitue le terroir, patrimoine naturel domestiqué et valorisé par l'homme. Ce savoir-faire est le fruit à la fois d'observations empiriques, acquises de génération en génération, et d'une recherche permanente associée notamment au mode de conduite de la vigne, à l'aménagement, à la gestion des sols et des coteaux. Parallèlement à la dimension purement viticole, les paysages s'enrichissent très souvent d'autres composantes, esthétiques en liaison avec le milieu naturel, l'architecture des domaines et des villages viticoles. En complément, la protection de la biodiversité locale s'intègre également dans une démarche d'Eco-Oenotourisme et participe souvent à la valorisation du paysage local (www. biodivine.eu). Au-delà des terroirs, l'éco conception des caves (www.ecowinery.eu) participe également à la valorisation écologique de la filière. Celle-ci repose sur l'optimisation de la gestion de l'eau et de l'énergie par l'utilisation de techniques de construction traditionnelle ainsi que de dispositif de pilotage. Inertie thermique du sous-sol, énergie solaire, murs et toits végétalisés, matériaux de construction écologique et harmonie paysagère s'intègrent dans une vision durable de la conception des caves. Au-delà des démarches individuelles, ces nouvelles thématiques justifient le plus souvent une réflexion et des démarches collectives associées à gouvernance à la fois écologique et patrimoniale des territoires viticoles. Cette vision est développée dans le cadre d'un projet « agriculture et paysage $\gg$ (http://www.vignevin.com/recherche/developpement-durable/paysages-viticoles/ agriculture-et-paysage.html) de la Charte Internationale « paysage viticole 》 (www.chartede fontevraud.org) qui souligne la mobilisation indispensable des différents acteurs du territoire (organisations viticoles, collectivités territoriales, office du tourisme et experts locaux) dans une approche pluridisciplinaire.
\end{abstract}

\begin{abstract}
Wine tourism has a growing share in the valuation of wine territories. This is also a factor which contributes to the image and the added value of the wines from these regions. Alongside the cultural attraction for the vine, wine and tasting, the "Wine tourism" is often motivated by an ecological approach cellars and vineyards. Thus emerges the concept of "Eco-tourism" which associates particularly in connection with sustainable development, landscapes, biodiversity and eco-design cellars. This topic is important for growers who are sensitive to the way society deals with their profession or for the acceptance of their activities and the projects they want to implement. Beyond the purely descriptive approach, the landscape is the aesthetic expression of the cultural ecosystem that is the soil, natural heritage and valued domesticated by man. This expertise is the result of both empirical observations acquired from generation to generation, and ongoing research, particularly associated with the driving mode of the vine, development, management of soils and slopes. Alongside the purely wine dimension, landscapes enrich often other components, aesthetic in connection with the natural environment, architecture domains and wine villages. In complement, protection of local biodiversity is also part of a process of Eco-Tourism and often participates in the enhancement of the local landscape (www. biodivine.eu). Beyond the land, eco design cellars (www. ecowinery.eu) is also involved in the ecological recovery of the sector. This is based on the immunization of the water management and energy by the use of traditional construction techniques as well as driving means. Thermal inertia basement, solar, green walls and roofs, green building materials and landscape harmony is part of sustainable vision of design cellars. Beyond the individual approaches, these new themes justify usually a reflection and collective approaches associated with governance in both ecological and heritage of wine territories. This vision is developed
\end{abstract}

\footnotetext{
${ }^{a}$ Auteur de correspondance : joel.rochard@vignevin.com
} 
within a French project "agriculture et paysage" (http://www . vignevin. com/recherche/developpementdurable/paysages-viticoles/agriculture-et-paysage.html) the framework of the International Charter "vineyard landscape" (www.chartedefontevraud.org) which highlights the vital mobilization of the various actors in the territory (wine organizations, local authorities, tourist and local experts) in a multidisciplinary approach.

\section{Introduction}

L'œnotourisme prend une part grandissante dans la valorisation des territoires viticoles. C'est également un facteur qui participe à l'image et à la valeur ajoutée des vins de ces régions. Parallèlement à l'attrait culturel pour la vigne, le vin et la dégustation, « l'Enotourisme » est souvent motivé par une approche écologique des caves et des terroirs (Fig. 1).

Ainsi émerge le concept 《d'éco-œnotourisme »qui associe notamment, en liaison avec le développement durable, les paysages, la biodiversité, ainsi que l'écoconception des caves. Le viticulteur et l'œnologue, au-delà de la production des raisins et de l'élaboration des vins, s'intègrent de plus en plus en la stratégie viticole, la promotion des terroirs, la conception et l'aménagement des caves. Dans sa mission d'ambassadeurs de l'Eco-œnotourisme, ils doivent veiller à mettre en œuvre les bases environnementales d'une viticulture et d'une œnologie durables, concept défini par une résolution de l'OIV (CST 01-2008).

« Approche globale à l'échelle des systèmes de production et de transformation des raisins, associant à la fois la pérennité économique des structures et des territoires, l'obtention de produits de qualité, la prise en compte des exigences d'une viticulture de précision, des risques liés à l'environnement, à la sécurité des produits et la santé des consommateurs et la valorisation des aspects patrimoniaux, historiques, culturels, écologiques et paysagers. \ [1] Cette thématique est importante pour les viticulteurs qui sont sensibles au regard que la société porte sur leur métier, voire pour l'acceptation locale de leurs activités et des projets qu'ils souhaitent mettre en œuvre [2-4].

Dans certains cas, la valorisation de leurs produits passe par le maintien de structures essentielles d'un paysage comme expression visuelle d'un lien au terroir (pour les produits avec une indication géographique par exemple) tel qu'il est défini par l'OIV (Viti 333/2010) : Le « terroir 》 vitivinicole est un concept qui se réfère à un espace sur lequel se développe un savoir collectif des interactions entre un milieu physique et biologique identifiable et les pratiques vitivinicoles appliquées, qui confèrent des caractéristiques distinctives aux produits originaires de cet espace. Le « terroir $»$ inclut des caractéristiques spécifiques du sol, de la topographie, du climat, du paysage et de la biodiversité.

Parallèlement les viticulteurs ont intérêt à offrir un paysage de qualité, support emblématique de l'activité touristique de leur région, vecteur d'image de leur métier et de leurs produits.

A propos de la valeur symbolique, émotionnelle, affective du terroir, S. MICHEL précise que «Nous sommes ce que nous mangeons. La méconnaissance de ce que nous avons dans notre assiette ou notre verre conduit à une perte d'identité, mais heureusement le

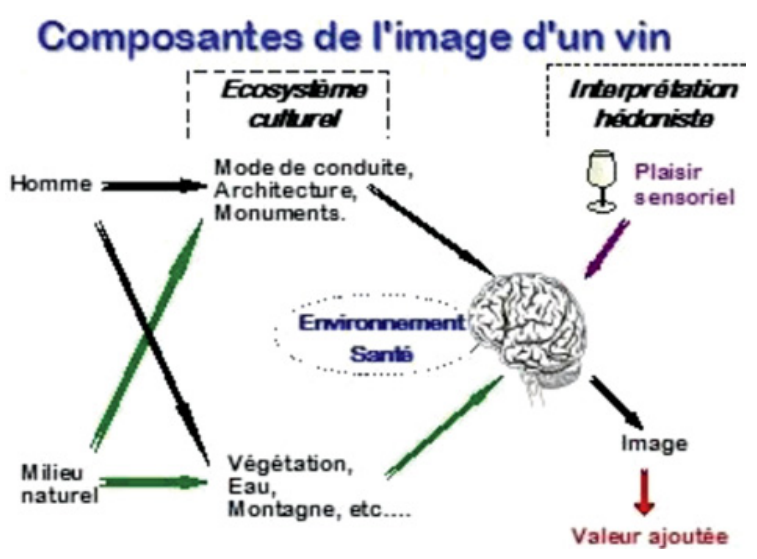

Figure 1. Composante de l'image d'un vin.

terroir restaure le lien entre le consommateur et l'aliment. Les valeurs symboliques de l'aliment sont celles du terroir mais attention, toute contamination du terroir ; qu'elle soit physique, chimique, biologique ou visuelle, contamine le produit dans l'esprit du client ».

Cette réflexion souligne que le terroir, et dans son prolongement la cave, sont des atouts, supports de valorisation, mais à l'inverse une dénaturation des paysages, ou une approche peu valorisante de la cave, peuvent dégrader la perception du terroir et indirectement de l'image du vin.

\section{Patrimoine et biodiversité des terroirs}

Par rapport au secteur agricole, le vin revêt une dimension culturelle voire intemporelle qui recouvre à la fois les sciences, les traditions, les savoir-faire ancestraux, les pratiques religieuses. Notre filière a imprimé une marque indélébile, une symbolique immortelle, dans l'évolution du monde et dans l'expression esthétique des terroirs. La beauté des paysages s'exprime dans de nombreux vignobles. Il existe de magnifiques paysages européens, traditionnels, souvent associés à un patrimoine bâti. Les pays émergents comportent également de très beaux paysages mis en valeur par un milieu naturel sauvage.

Au-delà de l'approche purement descriptive, le paysage est l'expression esthétique de «l'écosystème culturel $\gg$ que constitue le terroir, patrimoine naturel domestiqué et valorisé par l'homme. Cette dimension associe en premier lieu le savoir-faire technique du vigneron, à la fois architecte et jardinier, au sens noble du terme, du terroir. Ce savoir-faire est le fruit à la fois d'observations empiriques, acquises de génération en génération, et d'une recherche permanente associée notamment au mode de conduite de la vigne, à l'aménagement, à la gestion des sols et des coteaux. [5] Parallèlement à la dimension purement viticole, les paysages s'enrichissent très souvent d'autres composantes, 


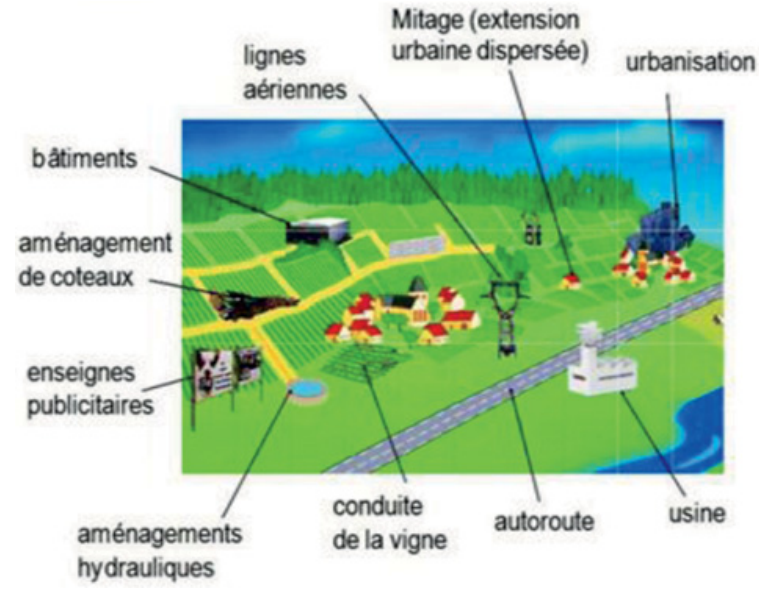

Figure 2. Facteurs de dégradation d'un paysage viticole.

esthétiques en liaison avec le milieu naturel, l'architecture des domaines et des villages viticoles [6].

Cependant, le paysage viticole est parfois menacé (Fig. 2). En premier lieu, l'arrachage, l'abandon ou l'urbanisation constituent des risques définitifs de disparition de panoramas remarquables. Des sources d'agressions complémentaires peuvent également dénaturer ces paysages (lignes électriques, aménagements routiers, éoliennes, panneaux publicitaires). Parallèlement aux aspects fonciers, l'urbanisation peut contribuer à la perte de conscience collective du patrimoine par effet de dilution de la population locale dans une communauté coutumière d'un mode de vie urbain.

En complément la biodiversité s'intègre également dans une vision durable du terroir. Le terme « biodiversité 》, contraction de 《diversité biologique »a été introduit au milieu des années 1980 par des naturalistes qui s'inquiétaient de la destruction rapide des milieux naturels et des espèces qu'ils hébergent. Une sensibilisation progressive des instances internationales aboutit à la signature en 1992 de la Convention sur la diversité biologique, lors de la conférence de Rio sur le développement durable. Elle définit la diversité biologique comme «la variabilité des organismes vivants de toute origine, y compris, entre autres, les écosystèmes terrestres, marins et autres écosystèmes aquatiques et les complexes écologiques dont ils font partie ». Dans une approche plus synthétique $\mathrm{C}$. Leveque précise que «la biodiversité est constituée par l'ensemble des êtres vivants, de leur matériel génétique et des complexes écologiques dont ils font partie ».

La biodiversité concerne plusieurs niveaux de systèmes biologiques :

- Diversité génétique : elle est caractérisée par l'ensemble de l'information génétique contenue dans tous les êtres vivants et correspond à la variabilité des gènes et des génotypes entre espèces et au sein de chaque espèce.

- Diversité spécifique (des espèces) : actuellement, la science recense environ 1,4 million d'espèces mais les naturalistes estiment que le potentiel de découverte est de l'ordre de 10 à 30 millions.

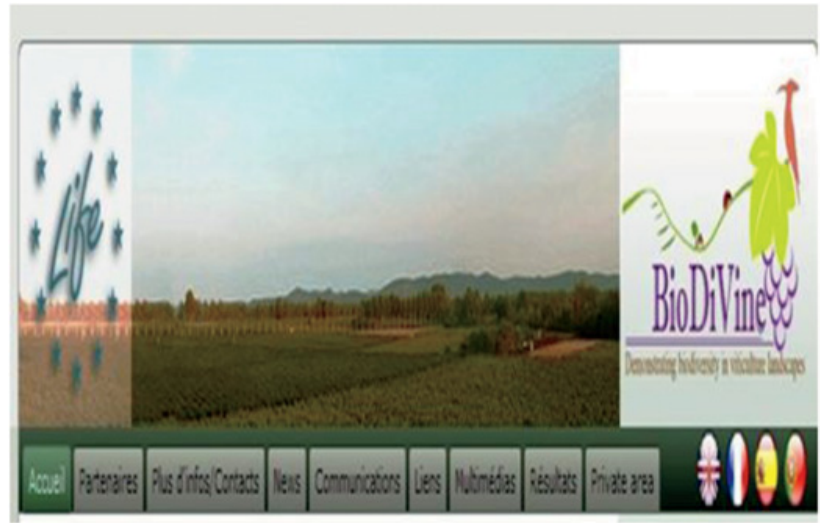

Figure 3. Projet européen BioDiVine.

- Diversité écosystémique (des écosystèmes) : le milieu naturel comporte de nombreux types d'écosystèmes interdépendants qui participent à l'équilibre biologique d'un territoire.

La prévention des ravageurs, maladies et plantes adventices, ainsi que la préservation des sols contre l'érosion, la salinisation, la contamination des sols par divers intrants sont autant de problématiques pouvant être considérées à différentes échelles. Bien que l'échelle de la parcelle ait toujours bénéficié d'un grand intérêt dans la recherche stratégique et appliquée, l'échelle supérieure plus globale du paysage a récemment connu un regain d'attention. En effet, la recherche interdisciplinaire sur des questions telle que la biodiversité fonctionnelle et la connectivité des éléments du paysage démontre que de nouvelles perspectives à l'échelle du paysage, dans une vision globale du terroir.

Depuis quelques années, de nombreuses expérimentations ont été menées pour optimiser les éléments seminaturels des territoires viticoles (arbres, haies, les bosquets, la couverture du sol, etc.. Les principales raisons invoquées pour l'amélioration de ces habitats sont les suivantes :

- Contribuer à la conservation de la nature (nourriture, abri, migration des habitats des plantes et animaux).

- Améliorer la qualité de l'environnement (bandes tampons pour limiter le transfert des pesticides, des métaux lourds et des éléments nutritifs). Promouvoir les valeurs esthétiques et patrimoniales des terroirs.

Le projet BioDiVine www . biodivine. eu porte sur l'étude et la gestion de la biodiversité dans les paysages viticoles (Fig. 3).

Ce projet, bénéficiant d'un financement européen LIFE + 2009/2014, visait à cerner l'intérêt des aménagements des terroirs viticoles et l'adaptation des itinéraires techniques concernant la biodiversité, le paysage et plus globalement l'environnement. Le projet se concentre sur la gestion de six sites expérimentaux européens (le Douro au Portugal et de la Rioja et Penedès en Espagne) et en France un site de référence, SaumurChampigny, les appellations de Saint-Emilion, Limoux Costières de Nîmes, la Bourgogne ainsi qu'un site associé (la Champagne). Des actions concrètes de conservation 


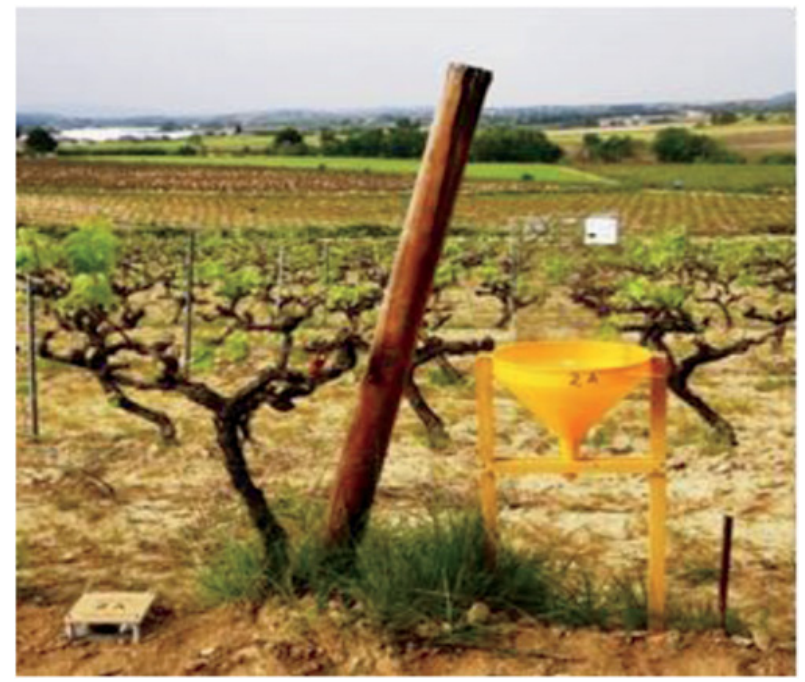

Figure 4. Evaluation de la biodiversité des arthropodes par la méthode RBA (Rapidity Biodiversity Assessment).

et d'aménagement ont été mise en œuvre sur chaque site avec la création d'espaces semi-naturels complémentaires tout en associant une protection raisonnée ou intégrée du vignoble. De plus, des suivis techniques par le biais de protocoles appropriés aux différentes spécialités de la biodiversité (Fig. 4) et une analyse cartographique ont été réalisés afin d'évaluer la portée de ces actions visant à conserver et augmenter la biodiversité.

Les aménagements paysagers favorables à la biodiversité doivent être interconnectés dans l'espace selon une stratégie réfléchie à l'échelle paysagère. A partir du lien entre les espaces semi-naturels déjà présents, les aménagements doivent être orientés à proximité des espaces les plus défavorables à la biodiversité afin de constituer des zones refuges interconnectés qui fournissent des abris et des ressources alimentaires aux arthropodes. Pour cela, les espaces inter-parcellaires, doivent donc être maintenus et si possible renforcés. Cet aspect concerne également les terrasses et les banquettes, qui représentent d'excellents supports de biodiversité dans les vignobles de forte pente. Les zones qui présentent une faible diversité floristique peuvent être optimisées par des semis plurispécifiques adaptés. Des ensemencements de mélange d'espèces adaptées peuvent être envisagés afin de diversifier la strate herbacée des nombreuses friches temporaires dont l'influence bénéfique sur la biodiversité peut être améliorée.

En complément, l'implantation de haies dans le vignoble de plaine, ou de faible pente, permet de rompre avec le paysage monocultural de type « mer de vigne » tout en augmentant le nombre d'habitats semi-naturels.

Parallèlement à leur effet bénéfique sur la biodiversité, ces aménagements présentent également des intérêts agronomiques. Par exemple, les bordures enherbées permettent de limiter la diffusion des pesticides hors de la parcelle grâce à leur pouvoir tampon en limitant les phénomènes de ruissellement/érosion. Concernant la protection du vignoble, les espèces végétales qui constituent les haies composites, judicieusement choisies peuvent constituer un refuge pour les communautés

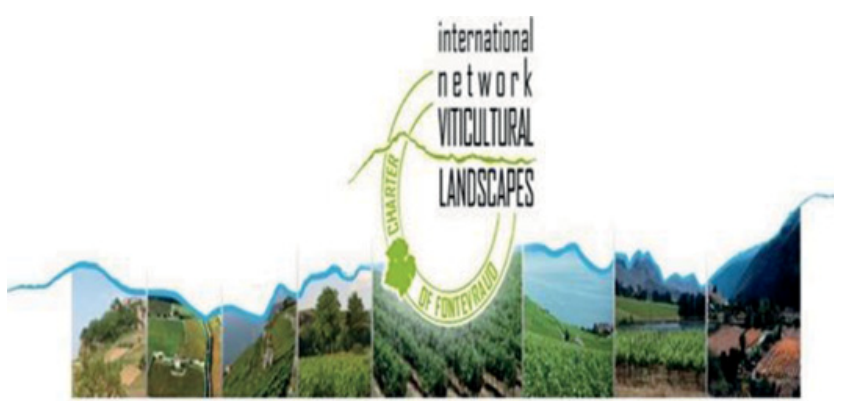

Figure 5. Réseau International de la Charte « Fontevraud ».

d'auxiliaires, facilitant ainsi la régulation naturelle des populations de ravageurs.

Il est important de considérer que le paysage ne doit pas être considéré comme un simple « sous-produit» des pratiques viticoles et territoriales, ce qui justifie de développer localement une « gouvernance patrimoniale et écologique » qui associe l'ensemble des acteurs du territoire (organisations viticoles, collectivités territoriales, office du tourisme et experts locaux) dans une approche pluridisciplinaire.

Cette gouvernance doit également associer la biodiversité viticole au sein du territoire. Entretien des murets de pierres sèches, plantation d'arbres et de haies, enherbement des parcelles, maintien de zones naturelles « réservoir » sont autant d'actions qui participent à l'équilibre de des écosystèmes associés aux vignobles. Dans ce cadre le Réseau International de la Charte Internationale Paysages Viticoles de Fontevraud www.chartedefontevraud.org a pour ambition d'inciter tous les acteurs des territoires viticoles, collectivités locales, syndicats viticoles, opérateurs de la culture et du tourisme, universités et laboratoires, à s'engager dans une gouvernance patrimoniale et durable du terroir. Elle intègre notamment des démarches paysagères volontaires et concertées conjuguant, dans une logique de développement durable, l'optimisation de la production viticole et la valorisation culturelle et touristique de ces paysages, au sein d'un réseau international d'excellence (Fig. 5).

Une 《boite à outil » a été développée pour cette démarche dans le cadre d'un projet mené en France «agriculture et paysage » http: //www. vignevin.com/ recherche/developpement-durable/paysagesviticoles/agriculture-et-paysage.html.

\section{Approche écologique des caves}

La construction d'un bâtiment viticole, et le choix des équipements associés, suppose une réflexion sur les aspects économiques, qualitatifs et de sécurité. Audelà de ces aspects fonctionnels, la notion d'écoconception des bâtiments se définit par la prise en compte du développement durable. Comme le souligne B. PEUPORTIER «l'éco-conception des bâtiments fait appel aux écotechnologies dans le domaine des économies d'énergies et d'eau, des énergies renouvelables (production d'énergie), de la gestion des déchets (matériaux et effluents), des matériaux à moindre impact, tout en incluant 
les aspects de confort, de santé et d'intégration paysagère. Architecture solaire, bioclimatique, solaire passif, haute qualité environnementale, basse consommation, énergie positive, zéro émission sont autant de termes usités pour définir des projets avant-gardistes intégrant les impératifs du développement durable. »

L'intégration du concept de développement durable au sein de la filière viticole associe, en premier lieu, une adaptation des itinéraires viticoles et œnologiques aux contraintes environnementales, mais également, avec une vision sur le long terme, une prise en compte des aspects énergétiques et de la gestion de l'eau dans la conception des exploitations et des caves. En effet, l'impact environnemental, lié à la conception des bâtiments et équipements associés, est assez faible comparativement aux itinéraires viticoles et œnologique mais, des choix liés à la construction, engagent l'exploitation et la cave pour plusieurs décennies.

Par ailleurs, il est probable qu'à l'image des normes de sécurité du personnel, la réglementation, les normes évoluent au cours des prochaines années ; ce qui justifie d'anticiper les exigences environnementales, afin d'éviter des modifications ultérieures de mise aux normes coûteuses.

D'une manière générale, l'optimisation des nettoyages, liée à la conception du matériel, du chai, ainsi qu'à l'organisation et à la sensibilisation du personnel, contribue à réduire les consommations, sans porter préjudice aux impératifs d'hygiène. L'expérience montre qu'une économie de 20 à $30 \%$ d'eau est rapidement obtenue à partir d'une réflexion interne et d'une sensibilisation du personnel. Les périodes de vendanges et de vinification génèrent une consommation journalière importante pouvant aboutir à une pénurie ponctuelle. Parallèlement, une gestion rationnelle de cette eau doit être envisagée pour limiter les pertes inutiles et faciliter le traitement d'épuration, dont le dimensionnement et les coûts de fonctionnement sont étroitement liés notamment au volume à traiter (ainsi qu'au niveau de pollution). Cet objectif justifie d'une part l'utilisation de méthodes de nettoyage et de désinfection appropriées et d'autre part une optimisation de la conception des chais. Par exemple, le choix de revêtement de sol suffisamment lisse, tout en évitant le risque de glisse, et la rationalisation des dispositifs d'écoulement, participent à une conception écologique des chais.

Le traitement des effluents s'intègre également dans la conception d'une cave. Concernant les caves, de nombreuses techniques ont été développées au cours des dernières décennies. Dans d'une vision de développement durable et d'éco conception de caves, il est important, au-delà des performances de l'épuration, d'intégrer plusieurs aspects : faible consommation d'énergie et limitation de l'impact sur l'effet de serre, production de déchets (boues) limitée, intégration paysagère et limitation de l'impact olfactif pour le voisinage. Les dispositifs basés sur l'utilisation de plantes et pures actrices (bambous, roseaux), utilisés seul ou en complément d'une technique classique, s'intègre dans cette vision durable (Fig. 6). Dans ce cadre il est également envisageable de contribuer à une valorisation énergétique des effluents (méthanisation,

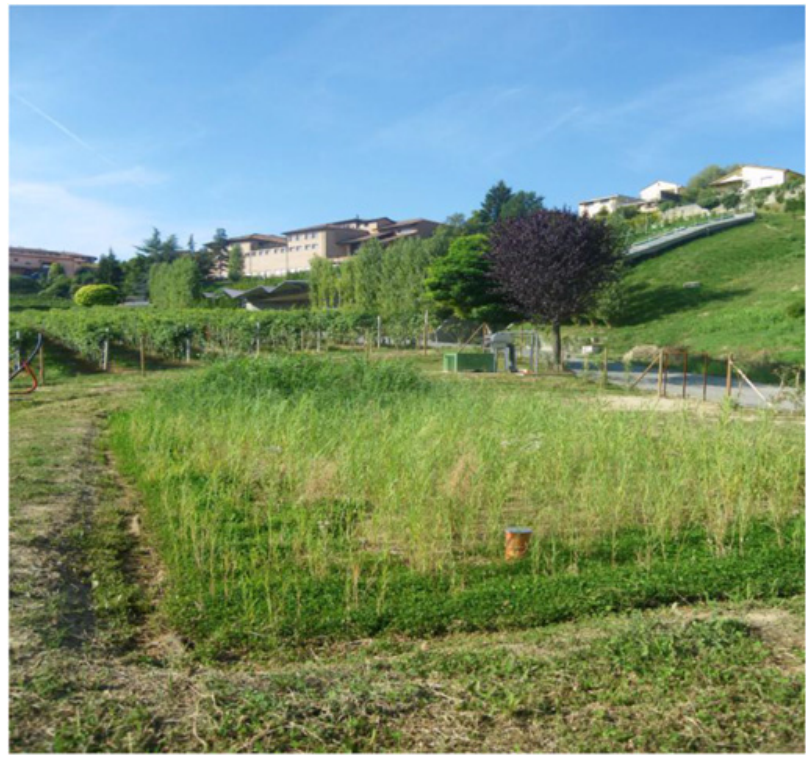

Figure 6. Dispositif de lits plantés de roseaux dans la région du Barolo en Italie.

compostage) en liaison avec la biomasse de la vigne et de la cave (sarments, marc, bourbes, lies).

Un projet européen ECOWINERY [7] www . ecowinery.eu a été développé afin d'apporter des informations aux professionnels de la filière sur l'éco conception des caves. Il a été réalisé dans le cadre du projet Leonardo Da Vinci financé par la Commission Européenne. Coordonné par l'Institut Français de la Vigne et du Vin, il regroupe des organisations de recherche/enseignement en France, Allemagne, Espagne et Suisse. Une plateforme Elearning a été créée sur les thèmes suivants : Contexte réglementaire, démarche architecturale et éco-construction, ressource énergétique associée au bâtiment (géothermie, solaire, pompe à chaleur, toit ou mur végétalisé), optimisation de l'utilisation de l'eau dans une cave vinicole, économie et gestion écologique des effluents, suivi et évaluation d'un projet d'éco-conception d'une cave vinicole et de son impact environnemental.

\section{Conclusion}

L'éco-œnotourisme s'intègre dans une stratégie durable d'une exploitation viticole et associe à la fois une vision écologique et patrimoniale. L'esthétique des terroirs témoigne de cette subtile harmonie que l'homme a su établir avec la nature. Les paysages-vignerons témoignent d'une diversité géologique unique et d'une histoire culturelle de la vigne et du vin sans égal. Mais le savoirfaire de l'homme ne s'arrête pas à la vigne. Au-delà du patrimoine des villages, des églises ou d'autres bâtisses historiques, l'architecture des caves, associées à une écoconception, participe pleinement à l'attractivité des régions viticoles.

Mais cet « écosystème culturel » est fragile. La modernité, dans ces excès, a souvent occulté ces richesses patrimoniales léguées par nos ascendants, patrimoine parfois perçu comme une contrainte face aux évolutions technologiques et aux impératifs économiques. Par 
ailleurs, il est probable que les variations climatiques à venir au cours des prochaines décennies, liées aux activités de l'homme, contribuent à perturber les particularités de nos terroirs.

Sachons protéger et transmettre à nos enfants cet héritage patrimonial, esthétique, écologique et communautaire, souvent mémoire d'intelligence, d'émotion et parfois de combat. C'est un capital qu'il convient de connaître, de préserver et de valoriser, si l'on veut perpétuer l'aspect culturel de la vigne, vecteur de la consommation du vin.

Les missions du viticulteur et de l'œnologue ont évolué au cours du temps. Si la maîtrise de la production des raisins et de l'élaboration des vins constituent toujours leur cœur de métier, les nouveaux enjeux de de la viticulture et de l'œnologie, s'intègrent progressivement dans leur savoir-faire, ce qui suppose notamment d'adapter les cursus de formation et les programmes de recherche/développement. Ainsi les dimensions écologiques, patrimoniales et culturelles de la filière viticole, supports de l'éco-œnotourisme, contribuent à enrichir la noblesse et la reconnaissance de leur métier, condition indispensable pour un «produit culturel » tel que le vin, dans un monde hyper médiatisé.

\section{Références}

[1] J. Rochard. Revue des œnologues et des techniques vitivinicoles et œnologiques. Application de la viticulture durable : Adaptations environnementales des itinéraires techniques. ISSN 0760-9868, 38, $\mathrm{N}^{\mathrm{o}}$. 138, pages 55-57 (2011)

[2] J. Rochard. Revue française d'œnologie. Du paysage à l'Eco-conception des caves : "L'onologue ambassadeur de l'eco-oenotourisme ». ISSN 0395-899X, $\mathrm{N}^{\circ}$ 252, pages 4-7 (2012)

[3] J. Rochard. Revue des œnologues et des techniques vitivinicoles et œnologiques. Eco-construction et développement durable. ISSN 0760-9868, 36, $\mathrm{N}^{\mathrm{o}}$. Extra 133, pages 11-12 (2009)

[4] J. Rochard. Le Progrès agricole et viticole. L'environnement dans le secteur viti-vinicole historique et perspectives. ISSN 0369-8173, 117, $\mathrm{N}^{\circ} 23$, pages 508-514 (2000)

[5] JP. Pigeat, M. Guillard, J. Rochard, N. Fourny. Les paysages viticoles : un enjeu du 2lème siècle pour la filière viticole. XXVème Congrès Mondial de la Vigne et du Vin : Paris 19-23 Juin/June/Junio 2000, 3, pages 55-62 (2000)

[6] J. Guenser, C. Forget, F. Planas, B. Porte, J. Rochard. Revue des œnologues et des techniques viti-vinicoles et œnologiques. Préserver la biodiversité au vignoble: Démonstration par le projet Life + BioDiVine dans le Limouxin. ISSN 0760-9868, 40, Nº 146, pages 9-11 (2013)

[7] J. Rochard, S. Penavayre et coll. Eco-conception des caves : du concept à la pratique, Projet européen Ecowinery. Congrès International de la Vigne et du Vin - Mendoza, Argentine (2014) 